\title{
AVALIAÇÃO ERGONÔMICA DE PULVERIZADORES COSTAIS UTILIZADOS NO SETOR FLORESTAL
}

Robson Shigueaki Sasaki², Marconi Ribeiro Furtado Júnior², Elton da Silva Leite², Amaury Paulo Souza ${ }^{3}$, Mauri Martins Teixeira ${ }^{4}$ e Haroldo Carlos Fernandes ${ }^{5}$

\begin{abstract}
RESUMO - Os pulverizadores costais são amplamente utilizados no setor florestal. No entanto, existem equipamentos que podem ocasionar certo grau de estresse ao operador, acarretando-lhe danos à sua saúde. Assim, objetivou-se com este trabalho avaliar, ergonomicamente, quatro pulverizadores costais (termonebulizador, pneumático motorizado, costal manual e costal elétrico) quanto aos parâmetros nível de ruído, nível de vibração de corpo inteiro, gasto energético e esforço físico. Na avaliação de ruído, utilizou-se um dosímetro $01 \mathrm{~dB}$, comparando os resultados com o que estabelece a Norma NR-15. Para os níveis de vibração, utilizou-se um acelerômetro triaxial, ligado a um processador de sinal do tipo Maestro, sendo os dados comparados com as Diretrizes Europeias. Já para gasto calórico e esforço físico, usaram-se um analisador de gases metabólicos fabricado pela Medgraphics ${ }^{\circledR}$ modelo VO2000 e um monitor cardíaco da marca Polar. Observou-se que o equipamento costal manual e o elétrico não apresentaram problemas quanto ao nível de ruído. Já o pulverizador pneumático e o termonebulizador exibiram valores acima do recomendado pela Norma NR-15, com níveis de 99,97 e 94,33 dB(A), respectivamente. Da mesma forma, os equipamentos motorizados tiveram níveis de vibração elevados em comparação com os demais equipamentos, com vibração global (A8) de 2,09 $\mathrm{m} \mathrm{s}^{-2}$ para o termonebulizador e 2,15 $\mathrm{m} \mathrm{s}^{-2}$ para o pneumático. Quanto ao gasto energético, houve pequenas variações quanto ao tipo de equipamento utilizado, com gasto variando entre 3,0 e 3,96 kcal min-1.
\end{abstract}

Palavras-chave: Dispêndio energético; Vibração; NR-15.

\section{ERGONOMIC EVALUATION OF KNAPSACK SPRAYERS USED IN FORESTRY}

\begin{abstract}
Knapsack sprayers have been widely used in forestry. However, some equipment may cause different levels of stress for the operators, causing harm to their health. Therefore, this study aimed to evaluate ergonomically four knapsack sprayers (thermal sprayer, pneumatic motorized, hand sprayer and electric sprayer), regarding to noise parameters, body vibration level, energy expenditure and physical effort. For noise evaluation, a dosimeter $01 \mathrm{~dB}$ was used, and the results were compared with the regulatory norm NR-15. For vibration levels, we used a triaxial accelerometer connected to a signal processor Maestro type, and data were compared to the European directives. For caloric expenditure and physical effort, we used a gas metabolic analyzer manufactured by Medgraphics ${ }^{\circledR}$ model VO2000 and a heart monitor from Polar brand. We observed that the hand and electric knapsack sprayers did not present problems related to the noise level. However, the pneumatic motorized knapsack and the thermal sprayer, showed levels above than recommended by regulatory norm NR-15 with levels of 99.97 and $94.33 \mathrm{~dB}(\mathrm{~A})$, respectively. Similarly, the motorized equipment presented high levels of vibration compared to the other equipments, with global vibration (A8) of $2.09 \mathrm{~m} \mathrm{~s}^{-2}$ for the thermal sprayer and $2.15 \mathrm{~m} \mathrm{~s}^{-2}$ for the pneumatic. Regarding to energy expenditure, there were small variations between the kind of equipment used, with expenditure ranging from 3.0 to $3.96 \mathrm{kcal} \mathrm{min}^{-1}$.
\end{abstract}

Keywords: Energy expenditure; Vibration; NR-15.

\footnotetext{
${ }^{1}$ Recebido em 25.07.2013 aceito para publicação em 03.12.2013.

${ }^{2}$ Programa de Pós-Graduação em Engenharia Agrícola, Universidade Federal de Viçosa, UFV. E-mail: < robsonsasaki@yahoo.com.br>, <marconi_furtado@hotmail.com>e <eltonleite@gmail.com>.

${ }^{3}$ Departamento de Engenharia Florestal da Universidade Federal de Viçosa, UFV, Brasil. E-mail: <amaury@ufv.br>.

${ }^{4}$ Departamento de Engenharia Agrícola da Universidade Federal de Viçosa, UFV, Brasil. E-mail: <mauri@ufv.br>e < haroldo@ufv.br>.
} 


\section{INTRODUÇÃO}

Os plantios florestais em áreas de Cerrado são muito importantes no suprimento da demanda de madeira para os mais diversos fins, como lenha, carvão, postes, escoramentos, serraria e fabricação de papel (SILVA et al., 2004), além de ser um setor gerador de emprego e renda.

Da mesma forma que em culturas anuais, o sucesso no setor florestal depende de um bom planejamento com a máxima produção e os mínimos custos possíveis. Diversos aspectos definem a produção florestal, como o correto preparo do solo, a adubação, a adequada seleção da espécie, as condições edafoclimáticas e o controle de doenças, insetos e plantas daninhas. Entre esses fatores, destaca-se o controle de plantas daninhas, uma vez que prejudica o crescimento e o desenvolvimento da cultura (TIBURCIO et al., 2012).

Para se realizar o controle das plantas daninhas, o método mais utilizado é o químico, em razão da economia de custos com mão de obra, além de apresentar maior agilidade na obtenção de resultados, principalmente em áreas extensas (GONÇALVES et al., 2011). Diversos equipamentos são utilizados no controle químico, ganhando destaque o pulverizador costal manual devido ao baixo custo de aquisição do equipamento e a variabilidade de uso em diferentes condições operacionais (LOPES et al., 2011). Outros equipamentos como o costal elétrico, o costal pneumático e os termonebulizadores também têm sido empregados. Basicamente, as características que diferem tais equipamentos é o espectro de gotas, volume de pulverização, capacidade operacional, eficiência de controle e os aspectos ergonômicos.

No âmbito ergonômico, Lopes et al. (2011) avaliaram o esforço físico do operador ao utilizar um pulverizador costal manual, verificando que tal operação é classificada como moderadamente pesada. Cunha e Teodoro (2006) avaliaram o nível de ruído de diferentes equipamentos costais motorizados, incluindo dois pulverizadores, e verificaram nível de ruído de aproximadamente 104 $\mathrm{dB}$, o que demonstra a necessidade do uso de protetores auriculares. Furtado (2004), ao avaliar diferentes pulverizadores costais motorizados, quanto aos níveis de ruído e vibração, também observou valores elevados de ambas as variáveis. Apesar dos referidos trabalhos, nota-se ainda que existe carência de estudos relacionados a área.

Revista Árvore, Viçosa-MG, v.38, n.2, p.331-337, 2014
Assim, objetivou-se com este trabalho avaliar ergonomicamente quatro pulverizadores utilizados no setor florestal quanto aos níveis de ruído, vibração, gasto energético e esforço físico.

\section{MATERIAL E MÉTODOS}

O ensaio foi realizado na Universidade Federal de Viçosa, no setor de Ergonomia Florestal, pertencente ao Departamento de Engenharia Florestal. Avaliaramse ergonomicamente quatro pulverizadores costais: 1) Pulverizador Costal Motorizado - acionado por um motor ciclo Otto de dois tempos; 2) Termonebulizador - acionado por um motor ciclo Otto de dois tempos; 3) Pulverizador costal hidráulico - acionamento manual; e 4) Pulverizador costal elétrico - motor elétrico.

Os parâmetros ergonômicos avaliados foram nível de ruído próximo ao ouvido do operador, nível de vibração transmitido ao operador e avaliação do gasto energético do trabalho. O operador vestiu roupas apropriadas para realizar a operação de pulverização (EPI).

\subsection{Ruído}

O operador foi instrumentado com um dosímetro, Marca 01 dB e Modelo WED 007, instalados nas proximidades do seu ouvido direito. Na coleta de dados de ruído, tomaram-se os devidos cuidados quanto às condições ambientais, medindo-se tanto a temperatura quanto a velocidade do ar ambiente, que durante os ensaios foram inferiores a $30^{\circ} \mathrm{Ce} 5 \mathrm{~m} \mathrm{~s}^{-1}$, respectivamente.

O equipamento fornece como leitura final o nível de ruído equivalente, que consiste do nível ponderado sobre o período de medição. Os dados foram confrontados de acordo com os limites estabelecidos pela NR-15 (Legislação Brasileira de Atividades e Operações Insalubres).

\subsection{Vibração}

Realizaram-se as medições de vibração, no ponto que ocorre maior transmissão de vibração ao corpo do operador, que neste caso são as suas costas. Utilizou-se um acelerômetro triaxial alojado em uma placa metálica com bordadura de borracha, sistema semelhante ao utilizado em avaliação de vibração de assentos. O acelerômetro foi ligado a um medidor de vibração Maestro, modelo $01 \mathrm{~dB}$, devidamente configurado para a posição em pé e com o sensor de vibração posicionado nas costas do operador. O Maestro mede a aceleração RMS, 
ponderada, nas frequências de 5 a 1.500 Hz. O Maestro processa os dados provenientes do acelerômetro, calcula os valores RMS em cada eixo, bem como a vibração global (A8), que é a vibração normalizada para uma jornada de trabalho de $8 \mathrm{~h}$, e baseado nas Diretrizes Europeias 2002/44/CE, que determina o tempo de alerta e o tempo de exposição limite.

\subsection{Dispêndio energético e esforço físico}

O dispêndio energético foi obtido de forma direta, utilizando-se um analisador de gases metabólicos VO2000 (Aerosport Medical Graphics ${ }^{\circledR}$ ) devidamente calibrado, em que se analisaram as variáveis $\mathrm{VO}_{2}$ (consumo de oxigênio em mL/kg/min), $\mathrm{VCO}_{2}$ (produção de gás carbônico em $\mathrm{mL} / \mathrm{kg} / \mathrm{min}$ ), QR (quociente respiratório $=\mathrm{VCO}_{2} / \mathrm{VO}_{2}$ ) e gasto calórico $(\mathrm{kcal} / \mathrm{min})$. Com os dados da variável gasto energético (kcal/min), classificaram-se as operações de acordo com Couto (1995) (Tabela 1).

Os diferentes equipamentos também foram caracterizados indiretamente, quanto à carga cardiovascular de trabalho (CCV) (equação 1). Empregouse o método proposto por Apud (1989). Para obter os dados de frequência cardíaca, utilizou-se um monitor cardíaco da marca Polar.

em que:

$$
\mathrm{CCV}=\frac{(\mathrm{FCT}-\mathrm{FCR})}{(\mathrm{FCM}-\mathrm{FCR})} 100
$$

CCV = carga cardiovascular (\%);

FCT $=$ frequência cardíaca de trabalho (bpm);

FCM = frequência cardíaca máxima (220 - idade); e

FCR = frequência cardíaca de repouso (bpm).

Tabela 1 - Classificação do esforço físico de acordo com o dispêndio energético.

Table 1 - Classification of physical effort according to energy expenditure.

\begin{tabular}{cc}
\hline $\begin{array}{c}\text { Classificação do } \\
\text { esforço físico }\end{array}$ & $\begin{array}{c}\text { Dispêndio energético } \\
\text { (kcal min } \text { men }^{-1}\end{array}$ \\
\hline Leve & $<3,0$ \\
Moderadamente pesada & 3,0 a 5,1 \\
Pesado & 5,11 a 6,8 \\
Pesadíssimo & 6,81 a 8,5 \\
Extremamente pesado & $>8,5$ \\
\hline
\end{tabular}

Fonte: COUTO, 1995.

Source: COUTO, 1995
Em seguida, os valores foram comparados com a frequência cardíaca limite que corresponde a $40 \%$ da carga cardiovascular, e determinados pela equação 2 .

$$
\mathrm{FCL}=0,40(\mathrm{FCM}-\mathrm{FCR})+\mathrm{FCR}
$$

Quando a carga cardiovascular ultrapassar 40\% (acima da frequência cardíaca limite), é necessário reorganizar o trabalho, sendo determinado, de acordo com Apud (1989), o tempo de repouso (pausa) necessário por meio da equação 3 .

$$
\mathrm{T}_{\mathrm{t}}=\frac{\mathrm{H}_{\mathrm{t}}(\mathrm{FCT}-\mathrm{FCL})}{\mathrm{FCT}-\mathrm{FCR}}
$$

em que:

$\mathrm{Tt}=$ tempo de repouso; $\mathrm{e}$

$\mathrm{Ht}=$ tempo total de trabalho.

Com a frequência cardíaca, de acordo com Apud (1997), classificaram-se as operações de pulverização (Tabela 2).

\subsection{População amostral}

O ensaio foi realizado empregando-se um único operador, com o intuito de comparar os diferentes pulverizadores quanto aos parâmetros ergonômicos. O operador possuía a idade de 24 anos, 1,78 m de altura e peso de $75 \mathrm{~kg}$.

\subsection{Coleta de dados}

Os parâmetros do gasto energético e frequência cardíaca, inicialmente, foram obtidos em laboratório, na condição em que o operador se encontrava em repouso.

Tabela 2 - Classificação da atividade de acordo com o batimento cardíaco.

Table 2 - Classification of activity according to the heartbeat.

\begin{tabular}{cc}
\hline $\begin{array}{c}\text { Frequência cardíaca } \\
\text { média }(\mathrm{bpm})\end{array}$ & $\begin{array}{c}\text { Classificação da } \\
\text { atividade }\end{array}$ \\
\hline$<75$ & Muito leve \\
$75-99$ & Leve \\
$100-124$ & Moderadamente pesado \\
$125-150$ & Pesado \\
$>150$ & Extremamente pesado \\
\hline
\end{tabular}

Fonte: APUD, 1997.

Source: APUD, 1997.

Revista Árvore, Viçosa-MG, v.38, n.2, p.331-337, 2014 
Posteriormente, com o operador instrumentado com todos os sensores descritos, dirigiu-se a um campo aberto, plano e com o solo coberto por gramíneas (Cynodon dactylon). Todos os pulverizadores foram abastecidos com a capacidade máxima de líquido e de combustível. Os equipamentos dotados de motores a combustão interna foram operados na aceleração máxima do motor.

O experimento foi realizado em delineamento inteiramente casualizado, com quatro tratamentos, representados pelos diferentes tipos de pulverizadores. Para cada equipamento, o operador caminhou durante o período de 2 min até que se estabilizasse a cadência de trabalho, dando início à coleta de dados, que foi de 15 min para cada equipamento, em condições semelhantes de trabalho. A cada troca de equipamento, houve um intervalo de 15 min de descanso ao operador.

\section{RESULTADOS}

\subsection{Nível de ruído}

O pulverizador pneumático e o termonebulizador apresentaram níveis de ruído de 99,97 e 94,33 dB(A), respectivamente. Vilela et al. (2005) avaliaram o nível de ruído emitido por um pulverizador costal motorizado no controle de vetores, encontrando 97,5 dB(A), valor próximo dos encontrados neste ensaio.

Ao confrontar-se com a NR-15, observou-se que os equipamentos motorizados apresentavam nível de ruído acima do estabelecido na norma, que recomenda valores inferiores a $85 \mathrm{~dB}$ para uma jornada de trabalho de $8 \mathrm{~h}$ e sem o uso de protetor auricular. Ainda de acordo com essa Norma, sem o uso de protetor auricular o equipamento pneumático que apresentar nível de ruído próximos de $100 \mathrm{~dB}(\mathrm{~A})$, a máxima exposição diária a ele é de apenas $1 \mathrm{~h}$; para o termonebulizador, com valores próximos de $95 \mathrm{~dB}(\mathrm{~A})$, essa permanência é de 2 h.
Os demais pulverizadores costais hidráulicos não apresentaram problemas quanto ao parâmetro ruído, com valores inferiores a $60 \mathrm{~dB}(\mathrm{~A})$, atendendo aos padrões da NR-15.

\subsection{Nível de vibração}

Da mesma forma que o parâmetro ruído das máquinas, verificou-se que a vibração transmitida ao operador também foi maior em equipamentos motorizados (Tabela 3).

Na avaliação de vibração de corpo inteiro, quanto ao parâmetro saúde, trabalhando-se na posição em pé e posicionando-se o sensor nas costas do operador, de acordo com a Diretiva Europeia, o nível de segurança que não causa danos à saúde do operador, denominado nível de alerta de aceleração, é $0,5 \mathrm{~m} \mathrm{~s}^{-2}$. Ainda de acordo com as normas europeias, nas condições impostas o nível-limite de aceleração é de 1,15 $\mathrm{m} \mathrm{s}^{-2}$.

Observou-se que, aparentemente, todos os equipamentos avaliados apresentaram valores acima do especificado na Diretiva Europeia, em especial os equipamentos motorizados. De acordo com os resultados, o tempo de exposição de alerta para o termonebulizador e o pulverizador pneumático foi de aproximadamente 26 e 28 min, respectivamente. Para um tempo máximo de exposição de 2 h:17 m e 2 h:23 m.

\subsection{Dispêndio energético e esforço físico}

Neste estudo, verificou-se que o maior consumo de oxigênio foi quando se utilizou o equipamento pneumático, e o menor consumo foi observado ao utilizar o termonebulizador, com gasto energético de 3,96 e 3,00 kcal/min, respectivamente (Tabela 4).

O dispêndio energético nas operações de pulverização foi relativamente baixo, nas condições do ensaio. Vale ressaltar que o ensaio foi realizado

Tabela 3 - Níveis de vibração de corpo inteiro dos diferentes equipamentos costais. Table 3 - Levels of body vibration for different knapsack sprayers.

\begin{tabular}{|c|c|c|c|c|c|c|}
\hline \multirow[t]{3}{*}{ Equipamento } & \multicolumn{4}{|c|}{ Aceleração RMS $\left(\mathrm{m} \mathrm{s}^{-2}\right)$} & \multicolumn{2}{|c|}{ Tempo (h:m:s) } \\
\hline & \multicolumn{3}{|c|}{ Eixo } & \multirow[t]{2}{*}{ Global (A8) } & \multirow[t]{2}{*}{ Nível de alerta } & \multirow[t]{2}{*}{ Nível limite } \\
\hline & $\mathrm{X}$ & $\mathrm{Y}$ & $\mathrm{Z}$ & & & \\
\hline Termonebulizador & 0,53 & 2,15 & 0,52 & 2,15 & 00:26:03 & 02:17:53 \\
\hline Pneumático & 0,42 & 2,09 & 0,53 & 2,09 & $00: 28: 30$ & $02: 23: 55$ \\
\hline Costal manual & 0,53 & 1,58 & 0,45 & 1,58 & $00: 48: 28$ & 04:16:22 \\
\hline Costal elétrico & 0,56 & 1,59 & 0,41 & 1,59 & $00: 48: 01$ & 04:14:03 \\
\hline
\end{tabular}

Revista Árvore, Viçosa-MG, v.38, n.2, p.331-337, 2014 
Tabela 4 - Gasto energético dos diferentes pulverizadores costais. Table 4 - Energy expenditure of different knapsack sprayers.

\begin{tabular}{|c|c|c|c|c|c|c|c|}
\hline Equipamento & $\begin{array}{c}\mathrm{VO}_{2} \\
\left(\mathrm{~L} \mathrm{~min}^{-1}\right)\end{array}$ & $\left(\mathrm{mL} \mathrm{kg}^{-1} \mathrm{~min}^{-1}\right)$ & $\begin{array}{c}\mathrm{VCO}_{2} \\
\left(\mathrm{~L} \mathrm{~min}^{-1}\right)\end{array}$ & $\begin{array}{c}\mathrm{CO}, \\
\left(\mathrm{mL} \mathrm{kg}{ }^{-1} \min ^{-1}\right)\end{array}$ & RQ & $\begin{array}{c}\text { Dispêndio } \\
\text { Energético } \\
\left(\text { Kcal min }{ }^{-1}\right)\end{array}$ & Classificação \\
\hline Termonebulizador & 0,58 & 7,78 & 0,63 & 8,39 & 1,10 & 3,00 & $\overline{\text { Mod. Pesada }}$ \\
\hline Pneumático & 0,82 & 10,42 & 0,68 & 9,47 & 0,94 & 3,96 & Mod. pesada \\
\hline Costal manual & 0,67 & 8,98 & 0,54 & 7,17 & 0,81 & 3,25 & Mod. pesada \\
\hline Costal elétrico & 0,62 & 8,26 & 0,54 & 7,17 & 0,88 & 3,04 & Mod. pesada \\
\hline
\end{tabular}

em campo, em local plano e avaliado apenas um operador. Os valores obtidos servem para se ter a noção da atividade, podendo esse valor ser maior ou menor de acordo com as condições de trabalho impostas.

Observou-se que, utilizando o método direto, as operações de pulverização, independentemente do equipamento, foram consideradas "moderadamente pesadas”, de acordo com a classificação de Couto (1995). Já ao se utilizar o método indireto, pela metodologia de Apud (1997), os diferentes equipamentos foram classificados em "moderadamente pesados" a "pesados”. Os métodos são bem condizentes entre si, e neste estudo o método de Apud (1997) demonstrou ser mais rigoroso (Tabela 5).

Nesta pesquisa, verificou-se que, ao utilizar os equipamentos pneumático, costal manual e elétrico, com o aumento da frequência cardíaca houve incremento do consumo de oxigênio e do gasto calórico pelo operador. Já para o termonebulizador houve menor gasto energético e elevada frequência cardíaca. Tal fato pode estar associado a outros fatores ergonômicos já relatados, como o ruído e a vibração, que foram elevados para tal equipamento.

Ainda de acordo com Apud (1989), recomendam-se realizar pausas durante a operação de pulverização, e para o equipamento pneumático a recomendação é de que se realize uma pausa de 19 min a cada 41 min de trabalho. Para o equipamento costal elétrico, a pausa é de 6 min a cada 54 min trabalhados.

\section{DISCUSSÃO}

No aspecto ergonômico, o ruído é considerado um dos principais fatores que afetam a saúde e eficiência dos trabalhadores (AYBEK et al., 2010). Entre as consequências, Guedes et al. (2010) relataram perdas auditivas lentas e progressivas, zumbidos persistentes e incomodativos. Também, o ruído pode ocasionar fadiga, nervosismo, náuseas e descuido durante o trabalho.

Neste caso, os pulverizadores acionados por motor de combustão interna apresentaram elevados níveis de ruído, corroborando Cunha e Teodoro (2006). Recomenda-se nesse caso, ao utilizar tais equipamentos, o uso de protetores auriculares por parte dos operadores, para que não ocorram danos prejudiciais à sua saúde. No entanto, vale ressaltar que os protetores auriculares devem ser escolhidos de acordo com o modelo e a qualidade e em conformidade com as características do usuário. Rodrigues et al. (2006) relataram que o funcionamento de um protetor auditivo depende de suas características e das características fisiológicas e anatômicas do usuário.

Outro importante parâmetro ergonômico, relacionado às máquinas, é a transmissão de vibração ao operador. Vários distúrbios estão associados à vibração, como

Tabela 5 - Esforço físico na operação de pulverização pelo método indireto, utilizando diferentes equipamentos. Table 5 - Physical effort during spraying by the indirect method, using different equipment.

\begin{tabular}{cccccc}
\hline Atividade & Frequência & $\begin{array}{c}\text { CCV (\%) } \\
\text { cardíaca (bmp) }\end{array}$ & FCL (bmp) & Tr (min/hora) & $\begin{array}{c}\text { Classificação } \\
\text { da atividade }\end{array}$ \\
\hline Repouso & 64 & - & - & - & - \\
Termonebulizador & 133 & 52,67 & 116,4 & 15 & Pesada \\
Pneumático & 140 & 58,02 & 116,4 & 19 & Pesada \\
Costal Manual & 123 & 45,047 & 116,4 & 7 & Mod. pesada \\
Costal Elétrico & 122 & 44,27 & 116,4 & 6 & Mod. pesada \\
\hline
\end{tabular}


efeitos vasculares, neurológicos, articulares, músculos esqueléticos, entre outros efeitos (TEWARI; DEWANGAN, 2009). Bluthner et al. (2006) afirmaram que a vibração pode levar a sérios danos à espinha dorsal dos operadores. Minette et al. (2010) afirmaram que, quando ultrapassam os limites estipulados pelas diretrizes europeias, os efeitos sobre a saúde e a segurança dos trabalhadores poderão estar sujeitos a riscos especialmente sensíveis.

Ao utilizar os equipamentos costais, devido à alta vibração encontrada, é necessário realizar pausas programadas durante o trabalho, o que na prática corriqueira já acontece, indiretamente, no momento do reabastecimento; ou então, nos equipamentos motorizados, utilizar sistemas de amortecimentos mais modernos em locais estratégicos e que reduzam a transmissão de vibração ao operador. Furtado (2004) avaliou um pulverizador costal motorizado e, ao final, estabeleceu o tempo de exposição de aproximadamente 25 min, corroborando esse resultado.

Quanto ao gasto energético, é diretamente proporcional ao consumo de oxigênio pelo indivíduo. $\mathrm{O}$ dispêndio energético utilizando os diferentes equipamentos é relativamente baixo. A título de comparação, uma caminhada leve consome em torno de 3 a 5 kcal min ${ }^{-1}$, enquanto uma corrida pode chegar a $10 \mathrm{kcal} \mathrm{min}^{-1}$. Souza et al. (2001), avaliando o dispêndio energético em operações florestais, observaram que o gasto energético por um operador de motosserra foi igual a 6,07 kcal $\mathrm{min}^{-1}$.

Quando são detectados excessos de carga física, Minette et al. (2007) recomendam pausas para evitar a sobrecarga de trabalho. Lopes et al. (2011) avaliaram a carga física imposta pelo equipamento costal manual durante a pulverização de herbicidas, classificando a operação como “moderadamente pesada”, o que corrobora este estudo. Entretanto, esses autores constataram que não havia necessidade de pausas durante essa operação. Tudo isso diferentemente dos resultados deste trabalho, em que se recomendam as pausas principalmente em equipamentos motorizados.

De certa forma, conforme já relatado, essas pausas ocorrem naturalmente durante os abastecimentos, no entanto se recomenda que essas paradas sejam realizadas de forma programada e em local confortável, reduzindo os riscos à saúde do operador. Ao se realizar uma pulverização de campo, os diferentes fatores ergonômicos devem ser avaliados em conjunto, devendo ser programado um manejo adequado de acordo com o parâmetro que apresentar o maior problema em questão.

\section{CONCLUSÕES}

O pulverizador pneumático e o termonebulizador podem acarretar danos auditivos ao operador, visto que apresentaram elevados níveis de ruído.

Devem-se utilizar protetores auriculares quando a pulverização for realizada por equipamentos motorizados.

A vibração dos equipamentos motorizados foi elevada, podendo prejudicar a saúde do operador.

Na operação de pulverização utilizando equipamentos costais, recomendam-se pausas programadas, a fim de evitar a fadiga do operador e danos à sua saúde.

Houve pequena variação na demanda energética dos diferentes equipamentos, ou seja, entre 3,00 e 3,96 kcal $\min ^{-1}$.

O esforço físico durante a operação foi “moderadamente pesado” para os equipamentos hidráulicos e “pesado” para os motorizados.

\section{REFERÊNCIAS}

APUD, E. Guidelines on ergonomics study in forestry. Genebra: ILO, 1989. 241p.

APUD, E. Temas de ergonomia aplicados al aumento de la productividad de la mano de obra em cosecha forestal. In: SIMPOSIO BRASILEIRO SOBRE COLHEITAE TRANSPORTE FLORESTAL. 3., Vitória, 1997. Anais... Vitória: SIF, 1997. p.46-60.

AYBEK, A.; KAMER, H. A.; ARSLAN, S. Personal noise exposures of operators of agricultural tractors. Applied Ergonomics, v.41, p.274281, 2010.

BLÜTHNER, R. et al. On the significance of body mass and vibration magnitude for acceleration transmission of vibration through seats with horizontal suspensions. Journal of Sound and Vibration, v.298, n.4, p.627-637, 2006.

COUTO, H. A. Ergonomia aplicada ao

trabalho: o manual técnico da máquina humana. Belo Horizonte: Ergo, 1995. v.2. 382 p. 
CUNHA, J. P. A. R.; TEODORO, R. E. F. Avaliação do nível de ruído em derriçadores e

pulverizadores motorizados portáteis utilizados em lavouras de café. Bioscience Journal, v.22, n.3, p.71-77, 2006.

Furtado, M. A. F. Pulverizador costal motorizado: Aspectos ergonômicos (ruído e vibração) e desempenho operacional. 2004. 73f. Dissertação (Mestrado em Agronomia) - Universidade Estadual de São Paulo, Botucatu, 2004.

LOPES, E. S.; OLIVEIRA, F. M.; RODRIGUES, C. K. Determinação da carga física de trabalho na atividade manual de herbicida. Ambiência, v.7, n.2, p.329-337, 2011.

MiNETTE, L. J. et al. Avaliação da carga de trabalho físico e análise biomecânica de trabalhadores da carbonização em fornos tipo “Rabo-Quente”. Revista Árvore, v.31, n.5, p.853-858, 2007.

MINETTE, L. J. et al. Avaliação ergonômica do protótipo de um motocoveador hidráulico, utilizado em atividade de silvicultura florestal. Engenharia na Agricultura, v.18, n.6, p.488-495, 2010.

GONÇALVES, K. S. et al. Seletividade de herbicidas aplicados em pós-emergência em pinhão manso (Jatropha curcas L.). Revista Brasileira de Herbicidas, v.10, n.2, p.110120, 2011.
GUEDES, I. L. et al. Avaliação dos níveis de ruído e vibração na atividade de coveamento semimecanizado em região montanhosa. Engenharia na Agricultura, v.18, n.1, p.912, 2010.

RODRIGUES, M. A. G.; DEZAN, A. A.; MARCHIORI, L. L. M. Revista CEFAC, v.8, n.4, p.543-547, 2006.

SILVA, K. R. et al. Custos e rendimentos operacionais de um plantio de eucalipto em região de cerrado. Revista Árvore, v.28, n.3, p.361366, 2004.

SOUZA, A. P. et al. Avaliação do esforço físico despendido por trabalhadores em operações florestais no estado de Minas Gerais. Revista Árvore, v.25, n.3, p.353-360, 2001.

TEWARI, V. K.; DEWANGAN, K. N. Effect of vibration isolators in reduction of work stress during fild operation of hand tractor.

Biosystems Engineering, v.103, p.146-159, 2009.

TIBURCIO, R. A. S. et al. Controle de plantas daninhas e seletividade do Flumioxazin para eucalipto. Cerne, v.18, n.4, p.523-531, 2012.

VILELA, R. A. G.; MALAGOLI, M. E.; MORRONE, L. C. Trabalhadores da saúde sob risco: o uso de pulverizadores no controle de vetores. Revista Produção, v.15, n.2, p.263-272, 2005. 
